\title{
Reflets
}

Revue d'intervention sociale et communautaire

\section{Regards croisés Ontario-Québec : les services de soins de santé mentale des communautés de langue officielle en situation minoritaire de 1950 à nos jours}

\author{
Marie-Claude Thifault, Marie Lebel, Isabelle Perreault et Martin Desmeules
}

Volume 18, numéro 2, automne 2012

Les inégalités sociales de santé chez les communautés francophones en situation minoritaire (CFSM) au Canada

URI : https://id.erudit.org/iderudit/1013176ar

DOI : https://doi.org/10.7202/1013176ar

Aller au sommaire du numéro

Éditeur(s)

Reflets, Revue d'intervention sociale et communautaire

ISSN

1203-4576 (imprimé)

1712-8498 (numérique)

Découvrir la revue

Citer cet article

Thifault, M.-C., Lebel, M., Perreault, I. \& Desmeules, M. (2012). Regards croisés Ontario-Québec : les services de soins de santé mentale des communautés de langue officielle en situation minoritaire de 1950 à nos jours. Reflets, 18(2), 122-139. https://doi.org/10.7202/1013176ar
Résumé de l'article

Considérant qu'un Canadien sur neuf hospitalisé pour maladie mentale retournera à l'urgence moins d'un mois après sa sortie d'hôpital, il importe de documenter, dans une perspective socio-historique, les itinéraires « transinstitutionnels » des personnes souffrant de troubles psychiques. Nous proposons ici une note de recherche sur l'enquête que nous menons sur l'évolution des services de soins de santé mentale des communautés de langue officielle en situation minoritaire, en particulier, les communautés francophones de l'Est et du Nord ontarien ainsi que de la communauté anglophone de l'Ouest du Québec. Ces regards croisés entre deux régions francophones de l'Ontario et le Montréal anglophone visent une meilleure compréhension de l'impact des facteurs de « langue de service » et de " culture » sur la santé mentale des communautés francophones en situation minoritaire (CFSM).
Tous droits réservés (C) Reflets, Revue d’intervention sociale et communautaire, 2012
Ce document est protégé par la loi sur le droit d'auteur. L'utilisation des services d'Érudit (y compris la reproduction) est assujettie à sa politique d'utilisation que vous pouvez consulter en ligne. 


\section{Regards croisés Ontario-Québec : les services de soins de santé mentale des communautés de langue officielle en situation minoritaire de 1950 à nos jours}

Marie-Claude Thifault, Ph.D.

École des sciences infirmières, Université d'Ottawa

Marie Lebel, Ph.D.

Université de Hearst

Isabelle Perreault, Ph.D.

Chercheure postdoctorale en histoire,

École des sciences infirmières, Université d'Ottawa

Martin Desmeules, $M A$

Centre d'histoire des régulations sociales,

Université du Québec à Montréal ${ }^{1}$

\section{Résumé}

Considérant qu'un Canadien sur neuf hospitalisé pour maladie mentale retournera à l'urgence moins d'un mois après sa sortie d'hôpital, il importe de documenter, dans une perspective sociohistorique, les itinéraires "transinstitutionnels " des personnes souffrant de troubles psychiques. Nous proposons ici une note de recherche sur l'enquête que nous menons sur l'évolution des services de soins de santé mentale des communautés de langue officielle en situation minoritaire, en particulier, les 
communautés francophones de l'Est et du Nord ontarien ainsi que de la communauté anglophone de l'Ouest du Québec. Ces regards croisés entre deux régions francophones de l'Ontario et le Montréal anglophone visent une meilleure compréhension de l'impact des facteurs de "langue de service " et de "culture " sur la santé mentale des communautés francophones en situation minoritaire (CFSM).

Mots clés : services de santé mentale, désinstitutionnalisation, minorités linguistiques, Ontario, Québec

\section{Abstract}

Considering that one in nine Canadians hospitalized for mental illness returns to emergency within one month after leaving hospital, we are interested in documenting, from a socio-historical perspective, the "trans-institutional" itineraries of people suffering from psychiatric disorders. We are proposing here a research note from the study that we are conducting on the evolution of mental health care services specifically for Francophone communities in Eastern and Northern Ontario, as well as for an Anglophone community in western Quebec. These comparisons between French-speaking regions of Ontario and Anglophones in Montreal will contribute to a better understanding of the impact that factors of "language service " and "culture " have on the mental health of Francophone minority communities.

Key words : mental health services, deinstitutionalization, linguistic minorities, Ontario, Quebec

\section{Introduction}

Nous proposons, dans le cadre de ce numéro portant sur «Les inégalités sociales de santé chez les communautés francophones en situation minoritaire (CFSM) au Canada ", une note de recherche sur l'enquête que nous menons sur l'évolution des services de soins de santé mentale des communautés de langue 
"La recherche en cours s'inscrit dans une problématique complexe incluant le développement des connaissances sur la désinstitutionnalisation en santé mentale et la réintégration des psychiatrisés 2 au sein de la société canadienne entre les années 1950 et 2010. ... elle s'applique à cerner la place sociale des personnes souffrant de troubles psychiques, dans un contexte où la question de la langue est un enjeu crucial en santé. " officielle en situation minoritaire, en particulier les communautés francophones de l'Est et du Nord ontarien ainsi que de la communauté anglophone de l'Ouest du Québec. La recherche en cours s'inscrit dans une problématique complexe incluant le développement des connaissances sur la désinstitutionnalisation en santé mentale et la réintégration des psychiatrisés 2 au sein de la société canadienne entre les années 1950 et 2010. Il s'agit d'une analyse inédite, notamment en ce qu'elle s'applique à cerner la place sociale des personnes souffrant de troubles psychiques, dans un contexte où la question de la langue est un enjeu crucial en santé. De fait, l'exploration de la dimension linguistique en ce qui a trait aux soins de santé mentale nous parait d'une importance capitale dans la construction d'une analyse socio-historique du phénomène sociétal de la désinstitutionnalisation au Canada.Ainsi, notre enquête, se penchant tant sur la réalité des psychiatrisés que sur celle des ex-psychiatrisés vivant en situation minoritaire en Ontario et au Québec, participe à une lecture plus complète et nuancée de l'expérience "transinstitutionnelle " qui culmine avec la déhospitalisation [transition entre l'hôpital et les soins communautaires].

Bien que depuis une dizaine d'années maintenant les travaux scientifiques internationaux en santé mentale sont de plus en plus produits à la lumière du concept d'empowerment (Aayard, Caria et Loubières, 2011), nous estimons qu'une réflexion socio-historique sur l'évolution des services offerts aux utilisateurs de soins en santé mentale dans les communautés linguistiques en situation minoritaire est préalablement pertinente et nécessaire. Ils décrivent les différents usages de l'habitation, les différents rapports au chez-soi. En effet, dans une volonté de reconnaitre la capacité et le pouvoir d'agir des personnes présentant un trouble psychique, il faudrait aussi, nous semble-t-il, mieux définir et comprendre la logique des itinéraires de migrations qui leur sont imposés dans un contexte «transinstitutionnel » de déhospitalisation. Notre équipe entend ainsi participer à l'évaluation des défis que représente l'empowerment des patients avec troubles psychiques dans des contextes socio-politiques et socio-linguistiques minoritaires. 


\section{Contexte de la recherche}

"Traitements, relations familiales, services communautaires sont quelques-unes des thématiques au cour de nos travaux que nous voulons dans le cadre de ce projet explorer, cette fois, en comparant les minorités francophones et anglophones de deux provinces..."
Depuis près d'une quinzaine d'années, nous avons mené des études exhaustives sur différentes facettes contribuant à mieux cerner la réalité asilaire en mettant au premier plan l'expérience des psychiatrisés eux-mêmes pour ensuite tisser une mosaïque socioculturelle et politique qui repose sur les liens indispensables avec la famille et sur l'univers extra-asilaire. Nous avons tenté de dépasser les murs de l'asile pour mieux comprendre le passage entre l'ère de l'institutionnalisation et celle de la désinstitutionnalisation (Thifault et Perreault, 2012). Nous avons dévoilé une fascinante correspondance, tirée des dossiers médicaux des patients de l'Hôpital Saint-Jean-de-Dieu dont l'analyse révèle que l'absence de réseaux de solidarités familiales avait un impact tant sur l'encadrement thérapeutique que sur la durée de l'internement des patients psychiatriques (Cellard et Thifault, 2007). Nos travaux ont permis aussi d'étudier les soins offerts en milieu asilaire en s'intéressant au rôle des infirmières laïques et religieuses spécialisées en nursing psychiatrique formées par les Sœurs de la Providence, fondatrices de la première école d'infirmières en nursing psychiatrique au Québec (1912-1962) (Thifault, 2009; 2010). Traitements, relations familiales, services communautaires sont quelques-unes des thématiques au cœur de nos travaux que nous voulons dans le cadre de ce projet explorer, cette fois, en comparant les minorités francophones et anglophones de deux provinces, soit les francophones vivant dans l'Est et le Nord ontariens et les anglophones de Montréal. Ce projet de recherche sur les soins de santé mentale et leur évolution depuis plus d'un demi-siècle nous permettra d'explorer les relations avec la parentèle, les politiques publiques en santé mentale, le développement des thérapies, les réactions et perceptions sociales de la maladie mentale et les cadres systémiques qui sont mis en place en Ontario. Des regards croisés avec la communauté minoritaire anglophone de Montréal serviront avantageusement la comparaison entre deux provinces et deux contextes linguistiques et culturels différents. Cette recherche servira à comprendre 
l'évolution de l'intervention en santé mentale sur la longue durée, à mieux connaître les besoins de la clientèle actuelle, à donner un éclairage nouveau sur les services de santé et enfin à questionner les politiques mises en place jusqu'à ce jour afin de proposer de nouvelles pistes qui participeront à l'amélioration des services en santé mentale dans les communautés francophones en situation minoritaire.

\section{État de la question}

L'historiographie des dix à quinze dernières années, surtout centrée sur les déterminants socio-économiques fondateurs de l'asile et d'autres institutions de régulation sociale, fait maintenant plus de place au profil de l'aliéné, à sa provenance, à ses liens sociaux et familiaux. Elle tend maintenant à démontrer que de complexes interactions sociales, hors du contrôle du milieu asilaire, interviennent dans l'internement des aliénés (Moran, 2000; Porter et Wright, 2003). L'approche révisionniste basée sur l'analyse croisée des variables de genre et de classe, au cœur de l'ouvrage Sex and Seclusion, Class and Custody (Andrews et Digby, 2004), contribue à définir des profils plus nuancés de la population asilaire. À l'instar des liens entre le sexe et la classe sociale des individus internés, l'état civil soulève également des distinctions permettant de mieux définir certains stéréotypes de l'enfermement (Thifault, 2011). L'absence de réseaux de solidarités familiales, par exemple, révèle la vulnérabilité des aliénés et aliénées, tel que démontré dans l'essai Une toupie sur la tête de Cellard et Thifault (2007). Ce phénomène d'isolement et de coupure sociale, qui n'est pas sans rappeler le concept d' "isolation " a un impact tant sur l'encadrement thérapeutique que sur la durée de l'internement pour certains patients.

D'autres recherches produites par des spécialistes sur l'histoire du nursing en psychiatrie au Canada ont tenté d'illustrer le caractère spécifique, original et même parfois dangereux des soins infirmiers psychiatriques prodigués à une clientèle reconnue au tempérament bouillant, colérique, belliqueux ou lymphatique et 
aux réactions plutôt imprévisibles (Dooley, 2004; Thifault, 2009; Tipliski, 2004). Les infirmières laïques ou religieuses étaient appelées à intervenir dans des situations relativement périlleuses lors de l'administration des soins à des patients violents. On s'en doute, dans un contexte aussi particulier, de nombreux dangers et risques font partie du travail de l'infirmière psychiatrique. Celle-là même participant à l'application du traitement moral qui deviendra au fil des ans de l'occupation thérapie. Cette approche thérapeutique se développe dans le contexte particulier de l'entredeux-guerres au moment où la conception des soins du XIX ${ }^{\mathrm{e}}$ siècle basée sur le schème de charité converge vers des ambitions curatives et repose principalement sur des activités de réadaptation dans un processus de réinsertion sociale.

Les projets de désinstitutionnalisation, soit le phénomène d'intégration des malades psychiatriques au sein de la société, n'ont pas retenu l'attention de beaucoup d'historiens québécois jusqu'à présent. Les précurseurs de ce champ d'études au Québec sont notamment les sociologues Françoise Boudreau (1984), Henri Dorvil $(1988 ; 2005)$ et le psychiatre Hubert Wallot (1998; 2005). Fidèles aux analyses anti-institutionnelles, ces auteurs se sont surtout intéressés au mythe de l'asile "curatif " en définissant l'asile comme lieu de régulation sociale et avant tout comme lieu d'abandon et de déshumanisation. Alors que sont publiées des études sur les francophones au Québec et sur les anglophones des autres provinces canadiennes, le sort des expsychiatrisés francophones et anglophones vivant en situation linguistique minoritaire au Canada demeure à découvrir. Comme en témoignent l'historiographie récente et les études sur la Colombie-Britannique (Menzies et Palys, 2006) et la Nouvelle-Écosse (Fingard et Rutherford, 2006) dans l'ouvrage Mental Health, c'est plutôt l'expérience, au cours des années 1950, des patients autochtones et anglophones hors Québec qui est révélée. Or, l'importance de la variable linguistique, de la classe sociale et de la culture de même que des représentations sociales des services en santé mentale aux communautés en situation minoritaire sont essentielles pour une meilleure compréhension de l'influence de ces déterminants sur l'institutionnalisation 
"Hors Québec, nous devons au sociologue Nérée St-Amand (2001; 2006; 2008) une contribution originale et sensible aux pratiques alternatives en santé mentale et cela tant sur les territoires de l'Ontario que du NouveauBrunswick. " ou la désinstitutionnalisation psychiatrique. Quant aux très intéressants travaux de Erika Dyck $(2008 ; 2009)$ sur un moment fort important lié à la prémisse de la désinstitutionnalisation, soit l'usage de drogues, ils présentent l'univers des Prairies et sa population anglophone.

Hors Québec, nous devons au sociologue Nérée St-Amand $(2001 ; 2006 ; 2008)$ une contribution originale et sensible aux pratiques alternatives en santé mentale et cela tant sur les territoires de l'Ontario que du Nouveau-Brunswick. Ses travaux sur la spiritualité dans le champ du travail social ouvrent la porte à un questionnement des liens entre l'État et l'Église qui marque indéniablement une remise en question des pratiques religieuses et, par conséquent, une relecture, également amorcée par les chercheurs en sciences humaines du Québec (Meunier, 2003; Gauvreau, 2005), sur la religion et la place qu'elle a occupée dans la prise en charge des aliénés des $\mathrm{XIX}^{\mathrm{e}}$ et $\mathrm{XX}^{\mathrm{e}}$ siècles. En définitive, c'est bien au Québec que le champ francophone de la désinstitutionnalisation est le mieux documenté. Dorvil (1988; 2005), spécialiste de l'expérience "transinstitutionnelle " au Québec, s'intéresse plus spécifiquement à la situation précaire des malades mentaux au sein de la communauté depuis les réformes sociales de la Révolution tranquille. Ses travaux ont grandement inspiré de nombreux sujets de thèses en histoire, en sociologie et en travail social (Duprey, 2007; Gagnon,1996; Morin,1994;Pitre, 1999; Robert, 1997). Néanmoins, la désinstitutionnalisation demeure un sujet qui a été, jusqu'ici, principalement étudié selon des perspectives sociologiques (Blais, Mulligan-Roy et Camirand, 1998; St-Amand, 2001; 2006). À ces études s'ajoutent les témoignages percutants des survivants de l'ère asilaire. Des récits qui, tout différents qu'ils soient les uns des autres, constituent des sources non négligeables pour la construction de la trame historique d'un phénomène sociétal, éclaboussé de scandales, et une meilleure compréhension du passé asilaire de personnes psychiatrisées (Pagé, 1961; Roy, 1994; St-Amand et Leblanc, 2008; Vigneault et Blais, 2007). L'éclatement des dispositifs de santé mentale, en particulier, au sein des communautés linguistiques en situation minoritaire demeure une thématique, jusqu'à ce jour, peu étudiée dans l'historiographie canadienne. 


\section{Approche socio-historique, 1950-2010}

"...l'approche

méthodologique que

nous privilégions

s'appuie, notamment,

sur la théorisation

ancrée ou la

grounded theory

(GT) qui allie

l'analyse empirique et

la conceptualisation de

catégories d'analyse. "
À la lumière de cet état de la question, l'approche méthodologique que nous privilégions s'appuie, notamment, sur la théorisation ancrée ou la grounded theory (GT) qui allie l'analyse empirique et la conceptualisation de catégories d'analyse. Cette approche insiste sur les discours des acteurs sociaux sans négliger le contexte (Laperrière, 1997). Particulièrement valide en sociologie et en histoire de la santé mentale, la GT propose une ouverture "d'écoute " propice à de nouveaux questionnements émergeant des données du terrain, sans hypothèse a priori, en insistant sur les perspectives des acteurs sociaux dans la définition de leur univers social. En ce sens, la GT se juxtapose judicieusement au champ de l'histoire culturelle des sensibilités qui a ouvert la voie à l'exploration des dimensions privée et intime dans un processus de réintégration sociale des personnes présentant un problème de santé mentale. C'est par l'étude du discours des témoins du système de soins en santé mentale qu'il nous sera possible de mieux comprendre les dynamiques "transinstitutionnelles " en milieu psychiatrique. Nos enquêtes, entre autres, dans les archives hospitalières et les dossiers médicaux des patients permettront une relecture de la période de la désinstitutionnalisation. Une analyse socio-historique nous permettra aussi de mettre en relief un thème, soit les services de santé mentale dans trois régions spécifiques, sur la longue durée. Cette analyse devrait nous aider à saisir la nature des discours, des politiques et des changements qui ont légitimé certaines formes de prise en charge des personnes psychiatrisées selon l'époque et le lieu, facteurs qui permettent une approche critique et constructiviste. L'évolution des contextes qui forgent les perceptions et les comportements à l'égard de la folie et influencent les politiques de gestion des comportements relevant de la maladie mentale seront au centre de nos questionnements de recherche.

Pour les segments de la recherche dont le terrain d'enquête reste encore largement en friche - particulièrement le Nord de l'Ontario où la fondation d'institutions est relativement récente 
- la démarche est essentiellement exploratoire et qualitative. Elle consiste à consulter l'ensemble des rapports ministériels sur les orientations de services en santé mentale pour la province de l'Ontario depuis l'après-guerre; à relever, pour la zone, les dates de fondation des centres d'intervention et des hôpitaux ayant un mandat d'intervention en santé mentale; à noter la coïncidence de ces fondations avec les lignes directrices des politiques publiques et à vérifier s'il y a prise en compte de la donnée linguistique dans les services et les soins dispensés. Plus encore, ce défrichage du terrain comporte l'identification de témoins clés rendant possible de retracer des itinéraires individuels représentatifs d'une mouvance plus large, celle des patients appartenant à une minorité de langue officielle et ayant reçu des soins santé mentale dans une zone excentrée et majoritairement anglophone. Un répertoire d'intervenants et de centres d'intervention avec des précisions sur les périodes d'opération sera dressée. L'ensemble des informations recueillies est compilé dans une base de données.

\section{Regards croisés : les terrains d'enquête}

"Cette recherche entend développer des observations et des connaissances sur les services de soins en santé mentale des communautés francophones et d'une communauté anglophone en situation minoritaire.»
Cette recherche entend développer des observations et des connaissances sur les services de soins en santé mentale des communautés francophones et d'une communauté anglophone en situation minoritaire. Les objectifs visés sont de 1) tracer le parcours obligé des ex-psychiatrisés en identifiant les ressources familiales et/ou sociales développées à leur intention dans leur région; 2) montrer l'impact des savoirs psychiatriques sur l'évolution des traitements, en particulier l'usage des traitements psychopharmacologiques, sur les modes de prise en charge des psychiatrisés; 3) cibler les enjeux professionnels des intervenants dans un contexte de déhospitalisation psychiatrique et, 4) déterminer l'impact du courant néolibéral sur la transformation des politiques en matière de santé mentale et leurs conséquences sur la clientèle psychiatrisée.

À l'aube d'une nouvelle phase de désinstitutionnalisation, la santé mentale est un sujet qui retient l'attention. Partout au pays, 
les statistiques témoignent de sa perpétuelle actualité. Ainsi, « un Canadien sur cinq est touché par la maladie mentale au cours de sa vie, personnellement ou dans son entourage immédiat " (Service de santé Royal Ottawa). L'an dernier, plus de 12000 Canadiens atteints de troubles de santé mentale ont dû être hospitalisés plus d'une fois en un mois (Radio-Canada, Radio-Journal, 8 juin 2011). Notre enquête sur la déhospitalisation psychiatrique est menée dans la province de l'Ontario au sein de deux CFSM, celles de l'Est et du Nord ontariens. Une approche comparative avec la population anglophone de Montréal participe à une meilleure compréhension de l'impact des facteurs de "langue de service " et de «culture " sur la santé mentale des CFSM.

\section{L'est de 1'Ontario}

Le point de départ des itinéraires suivis par les patients avec troubles mentaux au début du $\mathrm{XX}^{\mathrm{e}}$ siècle, est The Eastern Hospital for the Insane situé à Brockville et communément appelé le «Brockville Asylum ». L'asile change de nom en 1929 pour celui de Ontario Hospital Brockville avant de devenir le Brockville Psychiatric Hospital. Cette institution anglophone admet depuis 1894 les patients francophones de la région sans obligation aucune d'offrir des soins en français. C'est en 1910, en Ontario, que le terme "asile ", caractérisé par des traitements coercitifs, est remplacé par celui d'hôpital, plus moderne, où des soins spécialisés en médecine mentale sont accessibles. Ce changement de mentalité, alors que les asiles de l'Ontario font face à un état d'encombrement de plus en plus critique, s'accompagne de l'idée d'établir ce que l'Honorable W.J. Hanna appela le half-way-house. Ainsi débutait le long défi d'intégration de soins de santé mentale sans le recours automatique à l'hospitalisation des patients. Après un demi-siècle à convaincre la population que les chances de guérison dépendaient de la vigilance à interner un patient dès l'apparition des premiers symptômes de troubles mentaux, on soutient maintenant que les traitements idéaux pour les personnes insanes sont ceux offerts hors des murs asilaires. Cette réalité devient possible au Brockville Asylum, en particulier, grâce à l'augmentation du nombre d'infirmières disponibles tant de jour que de nuit pour accueillir 
"Le terrain estontarien s'intéresse aussi à l'histoire de l'école d'infirmières de Brockville (1903-1971), afin de déterminer explicitement le rôle professionnel des infirmières dans cette transition de déhospitalisation psychiatrique." les patients désorganisés. Le terrain est-ontarien s'intéresse aussi à l'histoire de l'école d'infirmières de Brockville (1903-1971), afin de déterminer explicitement le rôle professionnel des infirmières dans cette transition de déhospitalisation psychiatrique.

C'est en 1953 que l'Hôpital St-Louis-Marie-de-Monfort, symbole identitaire important des Franco-Ontariens, se distingue comme étant, dans l'Est ontarien, un repère incontournable de la mise en place de services de santé en français. Toutefois, c'est avec l'ouverture de son département de psychiatrie, en 1976, que nous pouvons stratégiquement ancrer le point de départ de notre enquête sur le territoire est-ontarien. Le rayonnement du programme de santé mentale de l'Hôpital Montfort, à travers ses services communautaires et ses partenariats avec des organismes du milieu comme La Maison Fraternité d'Ottawa (1962) sont des pistes importantes pour tracer les itinéraires «transintitutionnels » des francophones avec des troubles psychiques sur le territoire de l'Est de l'Ontario.

\section{Le nord de l'Ontario}

La communauté francophone de l'Ontario comptait, en 2006, 582690 personnes. Près du quart de cette population (22,5\%) réside dans la région dont nous proposons l'étude, cette proportion était plus importante, allant jusqu'à représenter le tiers de la population française en Ontario selon les moments de la période étudiée. Cela dit, pour une grande partie du $\mathrm{XX}^{\mathrm{e}}$ siècle, et jusqu'à tout récemment, il s'agit d'une population largement ouvrière, occupée au travail minier et forestier. Les individus se regroupent soit dans de petites communautés très homogènes, isolées et éloignées (Hearst, Fauquier, Kapuskasing, Moonbeam) ou dans des petites villes régionales où ils doivent composer avec une majorité anglophone détentrice des entreprises et des savoirfaire techniques et professionnels. Les travaux du sociologue Donald Dennie (1989; 1990) de l'Université Laurentienne ont bien montré la situation socio-économique des francophones dans le nord de l'Ontario au $\mathrm{XX}^{\mathrm{e}}$ siècle. Ce terrain d'enquête se penche sur les rapports et dossiers qui font les recommandations et posent les lignes directrices depuis 1950, nous incluons donc 
"Le segment nordontarien amorce un défrichage, selon une perspective chronologique et diachronique, d'un terrain d'enquête encore peu exploré, soit celui des soins de santé mentale offerts aux francophones. Il procède à l'identification des alternatives à l'hospitalisation et à la prise en compte, ou non, de la réalité linguistique $d u$ territoire. ") ici l'analyse de documents de portée nationale et provinciale, mais notre attention porte essentiellement sur la zone étudiée, ce qui concerne la région et les francophones.

Il n'est pas exclu de proposer des études de cas très concrètes, portant par exemple sur quelques centres de services précis : Hearst (présence francophone importante et susceptible d'obtenir des services en français), Kapuskasing (quartier général des services de counselling qui desservent aussi Hearst et Smooth Rock Falls en termes de consultation, d'évaluation et de traitement psychiatriques), Cochrane et Timmins (hôpital régional) en passant par Smooth Rock Falls (désintoxication). Deux périodes devraient retenir notre attention : celle de 1950-1960, moment où sont ouverts, dans la région immédiate, des centres de santé mentale (Sudbury et North Bay); et 1970-1990, moment où sont initiés des programmes de déhospitalisation et d'insertion communautaire (Association d'intégration communautaire, services de counselling, corridor du Nord, Services de toxicomanie, etc.) dans le milieu.

Le segment nord-ontarien amorce un défrichage, selon une perspective chronologique et diachronique, d'un terrain d'enquête encore peu exploré, soit celui des soins de santé mentale offerts aux francophones. Il procède à l'identification des alternatives à l'hospitalisation et à la prise en compte, ou non, de la réalité linguistique du territoire. Enfin, l'étude s'attarde à l'évolution de la communautarisation et il permet d'évaluer l'adaptation de celle-ci aux besoins linguistiques des patients francophones dans une perspective de longue durée.

\section{Montréal anglophone}

À l'ouverture de ses portes en 1890, le Verdun Protestant Hospital for the Insane (VPH) accueillait les aliénés et les patients chroniques de confessions protestantes provenant des institutions catholiques du Québec. Près d'un siècle plus tard, en 1977, le directeur médical du désormais Douglas Hospital intitulait son allocution au Bal de collecte de fonds des Auxiliaires-bénévoles « Bâtissons des ponts et non des murs ». Construits à la demande des philanthropes et commerçants anglo-montréalais pour soutenir les familles 
"D’une

institutionnalisation

intense des aliénés

au $X I X^{e}$ siècle, le $X X^{e}$ siècle sera

plutôt celui de la désinstitutionnalisation et du retour dans la communauté. Le parcours des psychiatrisés témoigne des changements de politiques et de pratiques autant que des réactions sociales face à ce qui relève de la déviance mentale. » et assainir le climat social de l'époque, le VPH connaît une rapide surpopulation. La politique d'internement hâtif de la fin du XIX ${ }^{\mathrm{e}}$ siècle, qui a pour but de traiter les aliénés dès les premiers symptômes, n'y est pas étrangère. Mais la Loi de 1909 du Gouvernement du Québec entend dissuader les proches d'interner l'un de leurs membres sans raisons « valables ", à savoir la dangerosité et les comportements antisociaux et scandaleux. D'une institutionnalisation intense des aliénés au $\mathrm{XIX}^{\mathrm{e}}$ siècle, le $\mathrm{XX}^{\mathrm{e}}$ siècle sera plutôt celui de la désinstitutionnalisation et du retour dans la communauté. Le parcours des psychiatrisés témoigne des changements de politiques et de pratiques autant que des réactions sociales face à ce qui relève de la déviance mentale.

Ce volet sur les parcours des psychiatrisés anglophones de la région du grand Montréal est d'autant plus important que très peu de recherches sur la longue durée ont été produites sur l'histoire des soins de santé mentale des Anglo-Montréalais (Paradis, 1997). Ce terrain d'enquête permet de rendre compte de parcours d'anglophones psychiatrisés en prenant l'exemple de l'un des trois principaux asiles québécois, le Verdun Protestant Hospital for the Insane, rebaptisé Verdun Protestant Hospital en 1925, et le Douglas Hospital en 1965. C'est dans les années 1950 qu'une politique de portes-ouvertes (open-door) est mise en place, parallèlement à l'établissement de programmes de soins en milieu familial, d'un service de consultation externe et de cliniques post-cures. La mi-temps du $\mathrm{XX}^{\mathrm{e}}$ siècle est également celle d'une révolution en matière de traitements psychiatriques avec l'introduction au VPH de la chlorpromazine en 1953 et de l'imipramine en 1957. Ces essais psychopharmacologiques auront, eux aussi, des effets considérables sur les nouvelles politiques de portes ouvertes.

En plus de ces nouveaux traitements, un élément majeur viendra bousculer l'institution psychiatrique à majorité anglo-protestante. Si, entre 1890 et 1962, la division des patients montréalais se fait selon la confession religieuse - les patients anglo-catholiques à Saint-Jean-de-Dieu et franco-protestants auVPH - c'est à partir de la Révolution tranquille que ces deux institutions accueillent désormais les patients sur la base de leur langue usuelle. En 1962, le Douglas Hospital devient officiellement un hôpital psychiatrique 
"Le Douglas

Hospital devient alors une institution psychiatrique bilingue contrairement à Saint-Jean-de-Dieu (rebaptisé Hôpital Louis-H.-Lafontaine en 1974) qui n'offrira que des services en français. » pour les anglophones de toutes confessions. Toutefois, dès 1970, la Commission Castonguay-Nepveu dépose son rapport qui ébranlera les structures administratives et linguistiques des institutions psychiatriques au Québec en proposant des services en fonction non plus de la langue mais du lieu de résidence. Le Douglas Hospital se verra desservir un secteur précis qui couvre le territoire Sud-Ouest de l'Île de Montréal. La loi 101 de 1977, proposée par le psychiatre Camille Laurin, sonne le glas à l'offre de soins unilingue anglophone de cette institution. Le Douglas Hospital devient alors une institution psychiatrique bilingue contrairement à Saint-Jean-de-Dieu (rebaptisé Hôpital LouisH.-Lafontaine en 1974) qui n'offrira que des services en français. Jusqu'à présent, aucune étude socio-historique des parcours «transinstitutionnels » des patients anglophones de Montréal n'a été réalisée. Cette recherche novatrice permettra de faire ressortir les enjeux contemporains des politiques québécoises en matière de santé mentale chez la population anglo-montréalaise et d'offrir des éléments comparatifs dans l'enquête sur les services offerts au franco-ontariens au cours de la même période.

\section{Discussion}

"L'identification des ressources offertes aux ex-psychiatrisés depuis 1950 nous permet d'avancer que la variable linguistique n'explique pas à elle seule l'accès et la qualité des services offerts dans les trois terrains d'enquête entre les années 1950 et 2010.»
Les résultats préliminaires de la recherche menée pour ce projet sur les services de santé mentale offerts dans les communautés linguistiques minoritaires à l'étude montrent que les situations different considérablement selon la province, la région et la langue. Les trois terrains d'enquête choisis sont à la fois porteurs de leur parcours socio-historique et de tensions politiques et économiques dans la mise en place de politiques publiques en santé mentale. L'identification des ressources offertes aux ex-psychiatrisés depuis 1950 nous permet d'avancer que la variable linguistique n'explique pas à elle seule l'accès et la qualité des services offerts dans les trois terrains d'enquête entre les années 1950 et 2010. Les communautés vivant en régions éloignées font face à un accès plus limité aux services que celles vivant en milieu urbain (Kulig et Williams, 2012). L'étude croisée des données entre le 
"...les institutions

et services en santé

mentale pour

les anglophones

montréalais sont

économiquement, structurellement et professionnellement mieux pourvus pour cause d'une tradition philanthropique bien établie dans cette communauté. » nord de l'Ontario, l'est de l'Ontario et le Montréal anglophone témoigne de disparités importantes. Par exemple, les institutions et services en santé mentale pour les anglophones montréalais sont économiquement, structurellement et professionnellement mieux pourvus pour cause d'une tradition philanthropique bien établie dans cette communauté. Aussi, la comparaison entre les services pour les francophones de l'est et du nord de l'Ontario donne matière à penser le développement plus soutenu des services aux francophones dans la région d'Ottawa, capitale fédérale, région qui a un poids démographique et politique plus important. L'analyse de la situation du nord de l'Ontario permet quant à elle de vérifier si la condition de fragilisation, qui est celle d'une minorité linguistique et culturelle, a été prise en compte au cours de la mise en place des centres et services d'aide aux patients souffrant d'une maladie psychique, dans une région excentrée déjà marquée par l'éloignement et les ressources limitées.

Cette recherche, qui couvre plus d'un demi-siècle, évalue la prise en charge et l'accès aux traitements psychiatriques (sociaux et chimiques) des personnes ayant des troubles de santé mentale. Elle se penche sur les enjeux professionnels de ce secteur de santé, sur l'évolution des ressources humaines disponibles, sur l'accessibilité selon la région (nombre de psychiatres, infirmières, travailleurs sociaux, éducateurs) et sur la congruence (ou l'incongruence) des programmes annoncés avec les décisions prises. L'étude offre une vue d'ensemble des changements de politiques publiques en matière de santé mentale au Québec et en Ontario, des déterminants sociaux de santé mentale et, enfin, des indicateurs développés en santé publique dans les trois régions à l'étude.

\section{Notes}

1 Marie-Claude Thifault (Ph.D. Histoire), professeure agrégée à l'École des sciences infirmières de l'Université d'Ottawa, directrice de l'Unité de recherche sur l'histoire des soins infirmiers et titulaire de la Chaire de recherche sur la francophonie canadienne en matière de santé. Marie Lebel (Ph.D. Histoire), professeure d'histoire à l'Université de Hearst. Isabelle Perreault (Ph.D. Histoire), professeure auxiliaire à l'École des sciences infirmières de l'Université d'Ottawa et post-doctorante à Concordia University. Martin Desmeules (MA Histoire), professionnel de 
recherche et coordonnateur du Centre d'histoire des régulations sociales de l'Université du Québec à Montréal.

2 Cet article ne s'intéresse aucunement au «jeu de langage », comme le dit Stiker, en ce qui concerne les manières sociales et culturelles acceptables de nommer toutes personnes présentant un handicap mental ou un trouble psychique. Considérant que le terme " usager " est anachronique dans le cadre de cette étude, nous avons privilégié l'utilisation d'une terminologie qui nous semble appropriée et fidèle au contexte historique auquel elle se rapporte.

\section{Bibliographie}

ANDREWS, Jonathan et Anne DIGBY (eds) (2004). Sex and Seclusion, Class and Custody : Perspectives on Gender and Class in the History of British and Irish Psychiatry, New York, Rodopi.

ARENDT, Hannah (2002). Aux origines du totalitarisme, suivi de Eichmann à Jérusalem, Édition établie sous la direction de P. Bouretz, Paris, Gallimard, Collection Quarto.

LAIS, Louise, Louise MULLINGAN et Claude CAMIRAND (1998). "Un chien dans un jeu de quilles : le mouvement des psychiatrisés et la politique de santé mentale communautaire en Ontario ", Canadian Review of Social Policy - RCPS,Vol. 42, p. 15-35.

BOUDREAU, Françoise (1984). De l'asile à la santé mentale. Les soins psychiatriques : histoire et institutions, $2^{\mathrm{e}}$ édition en 2003, Montréal, Éditions Saint-Martin.

CELLARD, André, et Marie-Claude Thifault (2007). Une toupie sur la tête. Visages de la folie à SaintJean-de-Dieu, Montréal, Boréal.

DENNIE, Donald (1990). "Le minoritaire franco-ontarien », Revue du Nouvel-Ontario, No 11, p. 181-183.

DENNIE, Donald (1989). "L'étude des réalités franco-ontariennes : à la recherche d'un nouveau modèle théorique», Revue du Nouvel-Ontario, No 1, p. 69-83.

DOOLEY, Chris (2004). « «They Gave Their Care, but We Gave Loving Care « : Defining and Defending Boundaries of Skill and Craft in the Nursing Service of a Manitoba Mental Hospital during the Great Depression ", Canadian Bulletin of Medical History / Bulletin canadien d'histoire de la médecine, Vol. 21, № 2, p. 229-252.

DORVIL, Henri (2005). «Nouveau plan d'action : quelques aspects médicaux, juridiques, sociologiques de la désinstitutionnalisation " Cahiers de recherche sociologique, $\mathrm{N}^{\text {os }} 41-46$, p. 209-235.

DORVIL, Henri (1988). Histoire de la folie dans la communauté, 1962-1987 : de l'Annonciation à Montréal, Montréal, Éditions Émile-Nelligan.

DUPREY, Catherine (2007). La crise de l'enfermement asilaire au Québec à l'orée de la révolution tranquille, mémoire de maîtrise en histoire, Université du Québec à Montréal.

DYCK, Erika (2009). «Prairies, Psychedelics and Place : The Dynamics of Region in Psychiatric Research ", Health and Place,Vol. 15, No 3, p. 888-894.

DYCK, Erika (2008). Psychedelic Psychiatry : LSD from Clinic to Campus, Baltimore, Johns Hopkins University Press.

FAYARD, Annick, Aude CARIA et Céline LOUBIÈRES (2011). "Dossier. Empowerment et santé mentale ", La santé de l'homme, Cedex, INPES, № 413, mai-juin, p. 7-44. 
FINGARD, Judith, et John RUTHERFORD (2008). Protect, Befriend, Respect. Nova Scotia's Mental Health Movement, 1908-2008, Halifax, Fernwood Publishing, p. 193-220.

FINGARD, Judith, et John RUTHERFORD (2006). «Social Disintegration, Problem Pregnancies, Civilian Disasters : Psychiatric Research in Nova Scotia in the 1950s " dans Mental Health and Canadian Society, Historical Perspectives, sous la dir. de James Moran et David Wright, Montréal/ Kingston, McGill-Queen's University Press, p. 193-220.

GAGNON, Brigitte (1996). Tout ce qu'on veut c'est vivre le plus normalement possible. Désinstitutionnalisation psychiatrique et communautarisation des pratiques en milieu franco-ontarien, mémoire de maitrise en service social, Université d'Ottawa.

GAUVREAU, Michael (2005). The Catholic Origins of Quebec's Quiet Revolution, 1931-1970, Montréal/ Kingston, McGill-Queen's University Press.

KULIG, Judith C., et Allison M.WILLIAMS (eds) (2012). Health in rural Canada, Vancouver, UBC Press.

LAPERRIÈRE, Anne (1997). "La théorisation ancrée (grounded theory) : démarche analytique et comparaison avec des approches apparentées " dans La recherche qualitative : enjeux épistémologiques et méthodologiques, Poupart et al. Montréal, Gaëtan Morin Éditeur.

MENZIES, Robert, et Ted PALYS (2006). «Turbulent Spirits : Aboriginal Patients in the British Columbia Psychiatric System, 1879-1950» dans Mental Health and Canadian Society, Historical Perspectives, sous la dir. de James Moran et David Wright, Montréal/Kingston, McGill-Queen's University Press, p. 149-175.

MEUNIER, Éric M. (2003). «Une nouvelle sensibilité pour les 'Enfants du Concile’? », dans Stéphane KELLY, Les idées mènent le Québec, Québec, Presses de l'Université Laval, p. 93-106.

MORAN, James (2000). Committed to the State Asylum, Insanity and Society in Nineteenth-Century Quebec and Ontario, Montréal et Kingston, McGill-Queen's University Press.

MORIN, Paul (1994). Espace urbain montréalais et processus de ghettoisation des populations marginalisées, thèse de doctorat en sociologie, Université du Québec à Montréal.

PAGÉ, Jean-Charles (1961). Les fous crient au secours, Montréal, Éditions du Jour.

PARADIS,André (1997). «Thomas J.W. Burgess et l'administration duVerdun Protestant Hospital for the Insane (1890-1916) ", Canadian Bulletin for the History of Medicine / Bulletin canadien d'histoire de la médecine, Vol. 14, № 1, 1997, p. 5-35.

PITRE, Jacynthe (1999). APPLE : Un visage parmi tant d'autres. Un modèle de pratique d'aide qui s'élabore "hors les murs " de l'institution pour les psychiatrisées, mémoire de maîtrise en service social, Université d'Ottawa.

PORTER, Roy, et David WRIGHT (2003). The Confinement of the Insane. International Perspectives, 1800-1965, Cambridge, Cambridge University Press.

ROBERT, Marie (1997). De la médicalisation à la pénalisation des justiciables souffrant de troubles mentaux, thèse de doctorat en sociologie, Université du Québec à Montréal.

ROY, Bruno (1994). Mémoire d'asile, Montréal, Boréal.

ST-AMAND, Nérée (2006). "Le sacré : au cœur ou en marge du social ", Reflets. Revue ontaroise d'intervention sociale et communautaire, Vol. 12, No 1, p. 20-47.

ST-AMAND, Nérée (2001). «Dans l'ailleurs et l'autrement : pratiques alternatives et service social ", Reflets. Revue ontaroise d'intervention sociale et communautaire, Vol. 7, No 2, p. 30-74. 
ST-AMAND, Nérée, et Eugène LEBLANC (2008). Osons imaginer. De la folie à la fierté, Moncton, OurVoice - Notre voix.

STIKER, Henri-Jacques, Corps infirmes et sociétés (2005 (1982)). Paris, Aubier.

THIFAULT, Marie-Claude (2011). «Aperçu historique. Les pratiques nursing dans le traitement des maladies nerveuses et mentales à l'Hôpital Saint-Jean-de-Dieu ", L'infirmière Clinicienne, revue francophone internationale. L'infirmière et la santé mentale, Vol. 8, No 2, p. 1-7.

THIFAULT, Marie-Claude (2010). «Le nursing psychiatrique à l'École de gardes-malades de l'Hôpital Saint-Jean-de-Dieu : 'le côté spirituel en tête du côté technique'", Scientia canadensis, vol. 33, no 1, p. 95-118.

THIFAULT, Marie-Claude (2009). «Au-delà d'un rôle de protection à l'égard des aliénés : initiation à l'art du nursing à l'Hôpital Saint-Jean-de-Dieu, 1912-1915 ", dans SanniYAYA (dir.) Pouvoir médical et santé totalitaire. Conséquences socio-anthropologiques et éthiques, Québec, Presse de l'Université Laval, p. 341-358.

THIFAULT, Marie-Claude, et Isabelle PERREAULT (2011). «Premières initiatives d'intégration sociale des malades mentaux dans une phase de pré-désinstitutionnalisation :l'exemple de SaintJean-de-Dieu, 1910-1950", Histoire sociale/Social History, Vol. 44, No 88, p. 197-222.

TIPLISKI,Veryl Margaret (2004). " Parting at the Crossroads : The Development of Education for Psychiatric Nursing in Three Canadian Provinces, 1905-1955 ", Canadian Bulletin of Medical History / Bulletin canadien d'histoire de la médecine,Vol. 21, No 2, p. 253-280.

VIGNEAULT, Luc, et Marcel BLAIS (2007).Témoignages dans la série Les maudits fous!, Montréal, Vendôme.

WALLOT, Hubert (2006). Peut-on guérir d'un passé asilaire? Survol de l'histoire socio-organisationnelle de l'hôpital Rivière-des-Prairies, Montréal, Publications MNH (premier tirage 2005).

WALLOT, Hubert (1998). La danse autour du fou : entre la compassion et l'oubli. Survol de l'histoire organisationnelle de la prise en charge de la folie au Québec depuis les origines jusqu'à nos jours (préface de Camille Laurin), Beauport, Publications MNH. 